\title{
Analysis of intrinsic peptide detectability via integrated label-free and SRM- based absolute quantitative proteomics
}

Andrew F Jarnuczak ${ }^{1}$, Dave C H Lee ${ }^{2}$, Craig Lawless ${ }^{1}$, Stephen W Holman ${ }^{3}$, Claire E Eyers ${ }^{3^{*}}$, Simon J Hubbard $^{1 *}$

1. Faculty of Life Sciences, University of Manchester, Michael Smith Building, Oxford Road, Manchester M13 9PT

2. Institute of Cancer Sciences, University of Manchester, 2nd Floor, Wolfson Molecular Imaging Centre, 27 Palatine Road, Withington, Manchester, M20 3JL

3. Centre for Proteome Research, University of Liverpool, Department of Biochemistry, Institute of Integrative Biology, Liverpool, L69 7ZB, UK

*Corresponding Authors:

Prof. Simon Hubbard; Faculty of Life Sciences, University of Manchester, Michael Smith Building, Oxford Road, Manchester, UK; simon.hubbard@manchester.ac.uk; +44 (0)1613068930

Prof. Claire Eyers; Centre for Proteome Research, Institute of Integrative Biology, University of Liverpool, Biosciences Building, Crown Street, Liverpool, UK; Claire.Eyers@liverpool.ac.uk; +44 (0)151 7954424

Author contact details:

Andrew F. Jarnuczak

Dave C Lee

Craig Lawless

Stephen Holman

Claire Eyers

Simon Hubbard email: andrew.Jarnuczak@manchester.ac.uk email: dave.lee-2@manchester.ac.uk email: craig.lawless@manchester.ac.uk email: stephen.holman@liverpool.ac.uk email: claire.eyers@liverpool.ac.uk email: simon.hubbard@manchester.ac.uk
Tel: +441612750000

Tel: +44 1612750000

Tel: +441612751680

Tel: +44 1517945344

Tel: +44 1517954424

Tel: +441613068930

\section{Supplementary Material}

Supplementary Figure S1. F-factor distributions in yeast proteins

Supplementary Figure S2. Properties of detected peptides in terms of F-factor classes and protein abundance.

Supplementary Figure S3. Ion series detected in peptide subsets classified by F-factor...... S4 Supplementary Figure S4. Peptide signal intensity/F-factor distributions filtered by missed cleavage prediction....

Supplementary Methods. S6

Supplementary References S6 


\section{Supplementary Figure S1. F-factor distributions in yeast proteins}

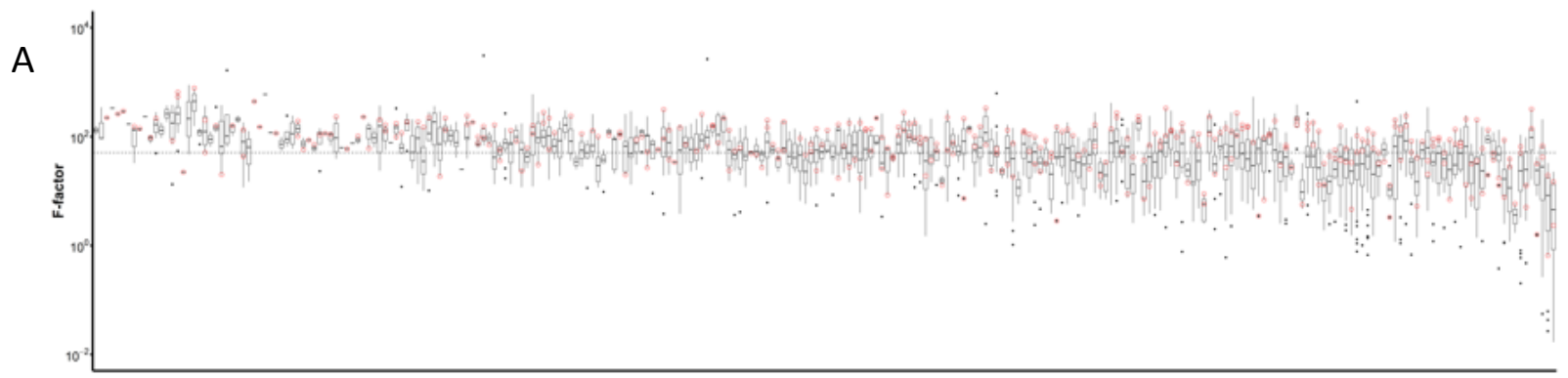

B

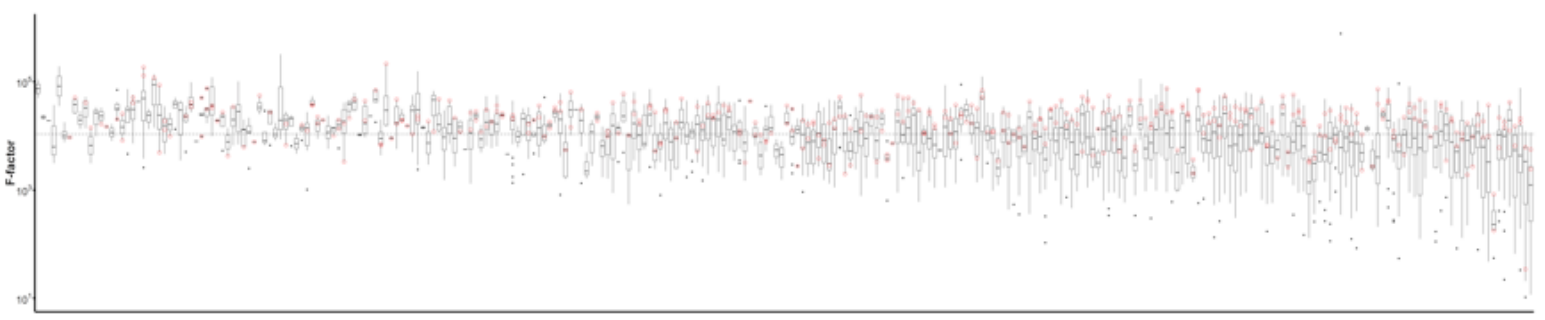

C

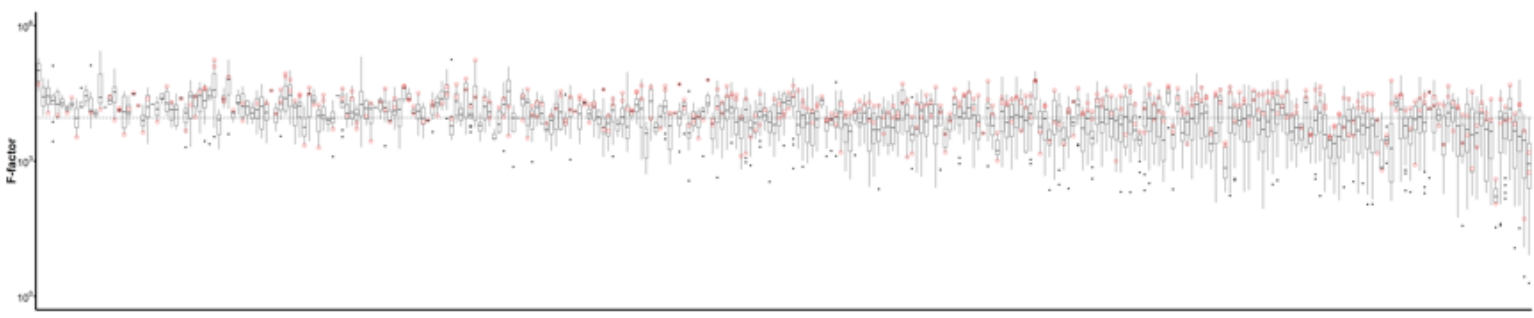

Supplementary Figure S1. F-factor distributions in yeast proteins. Distributions of yeast peptide F-factors for detected proteins from the 349 SIL-SRM-MS dataset, collected on A) LTQ orbitrap Velos on a 240 min gradient, B) Q Exactive HF under a 60 min gradient, and C) Q Exactive HF under a 120 min gradient. As in Figure 2, the median F-factor across all peptides is shown as a dotted line in both plots, and the F-factor values corresponding to the surrogate peptides used in the SRM experiments ( $\max =2$ per protein) are shown as red circles. Generally, these peptides are at the upper end of the F-factor distribution, illustrating the fact they were deliberately chosen as quantotypic peptides likely to possess good detectability. 
Supplementary Figure S2. Properties of detected peptides in terms of F-factor classes and protein abundance.
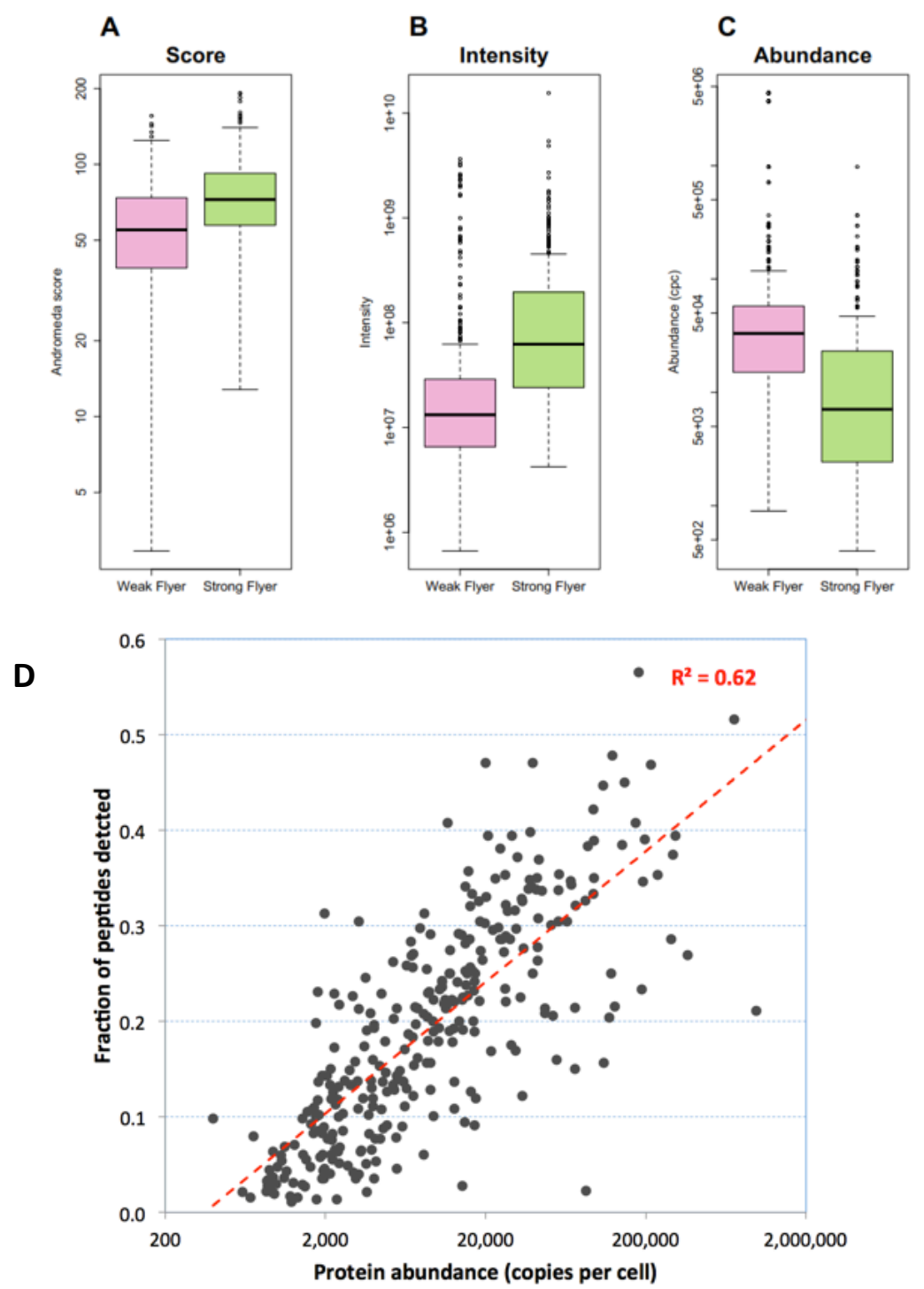

Supplementary Figure S2. Properties of detected peptides in terms of F-factor classes and protein abundance. Distributions of peptide and protein properties are shown in A-C for peptides classed as 'Weak' or 'Strong' flyers according to their F-factors, using the Q Exactive HF 120 minute gradient data. In A, the MaxQuant Andromeda search engine score distribution is shown for the two classes, highlight the improved quality of peptide-spectrum-match for strong flyers. In $B$, we note that strong flyers also tend to be those with high raw intensity as detected by the instrument. Similarly, the strong flyers are over-represented by proteins with low abundance (C). Finally, there is a good correlation between the fraction of peptides detected and protein abundance (D). These properties are consistent with the model illustrated in Figure 3, which shows how poorly ionising peptides will not be detected in low abundance proteins, thereby leading to the effects observed in $C$ and $D$ and presenting indirect evidence that Ffactors remain an independent, intrinsic measure that can be used independent of protein abundance, certainly for low abundance proteins. 
Supplementary Figure S3. Ion series detected in peptide subsets classified by F-factor
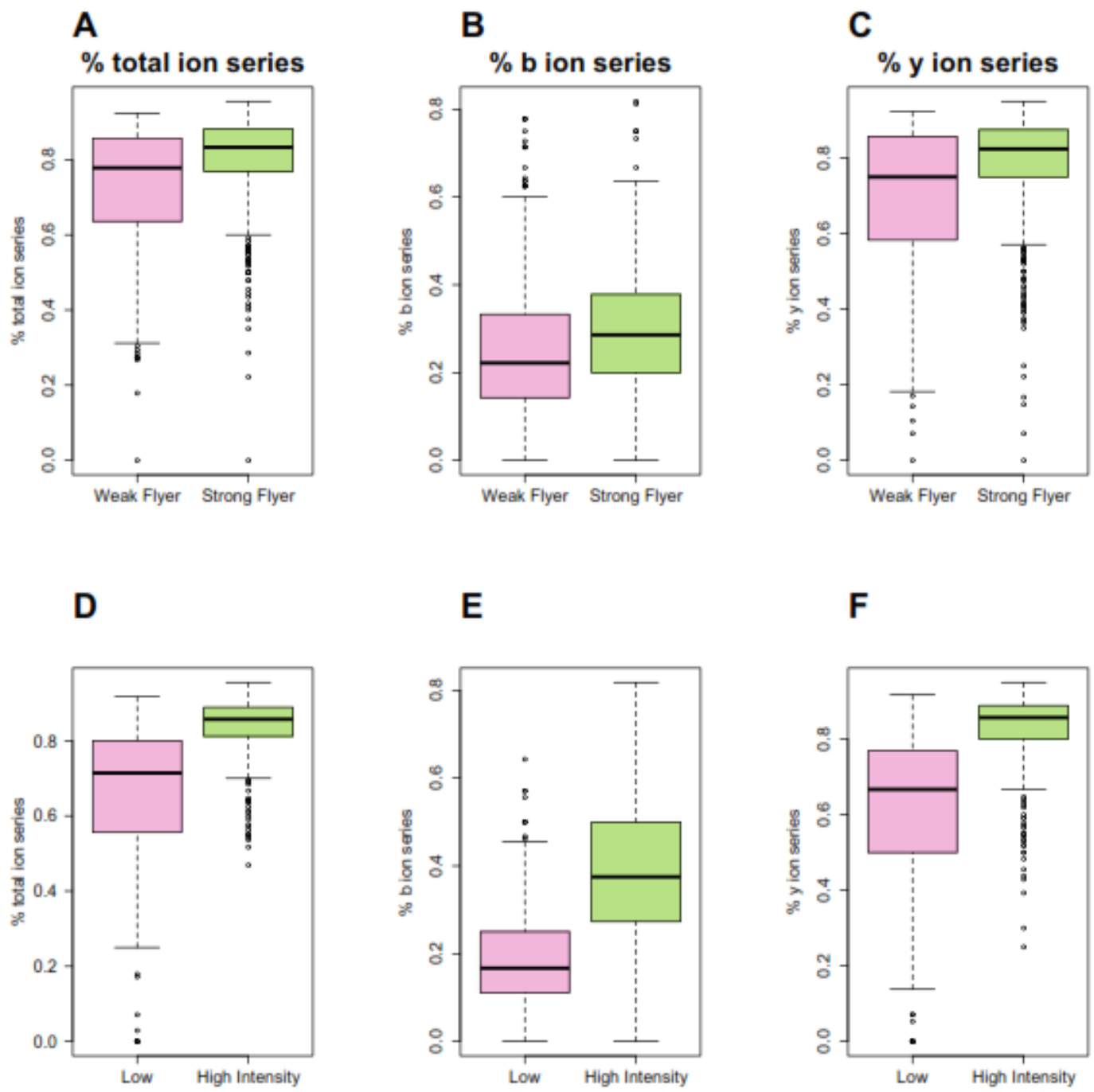

Supplementary Figure S3. Ion series detected in peptide subsets classified by F-factor. The detected ions reported by the Andromeda search engine were mapped back to the peptide Ffactor class, taking data from the most extensive experiment on the Q Exactive HF 120 min gradient. The plots in A-C show the fraction of total, $b$ and $y$-ions detected in the differing $\mathrm{F}$ factor classes, with corresponding plots for the raw intensity shown in D-F, defining intensity classes in the same way as F-factors with top/bottom $20 \%$-iles used to define classes. As might be expected the strong flyers are generally more complete in terms of fragmentation, as shown by a greater percentage of ion series detected. However, this effect is lessened in the F-factor classes compared to the intensity. This suggests that fragmentation, per se, does not have a large, direct and explicit effect on F-factors. 
Supplementary Figure S4. Peptide signal intensity/F-factor distributions filtered by missed cleavage prediction

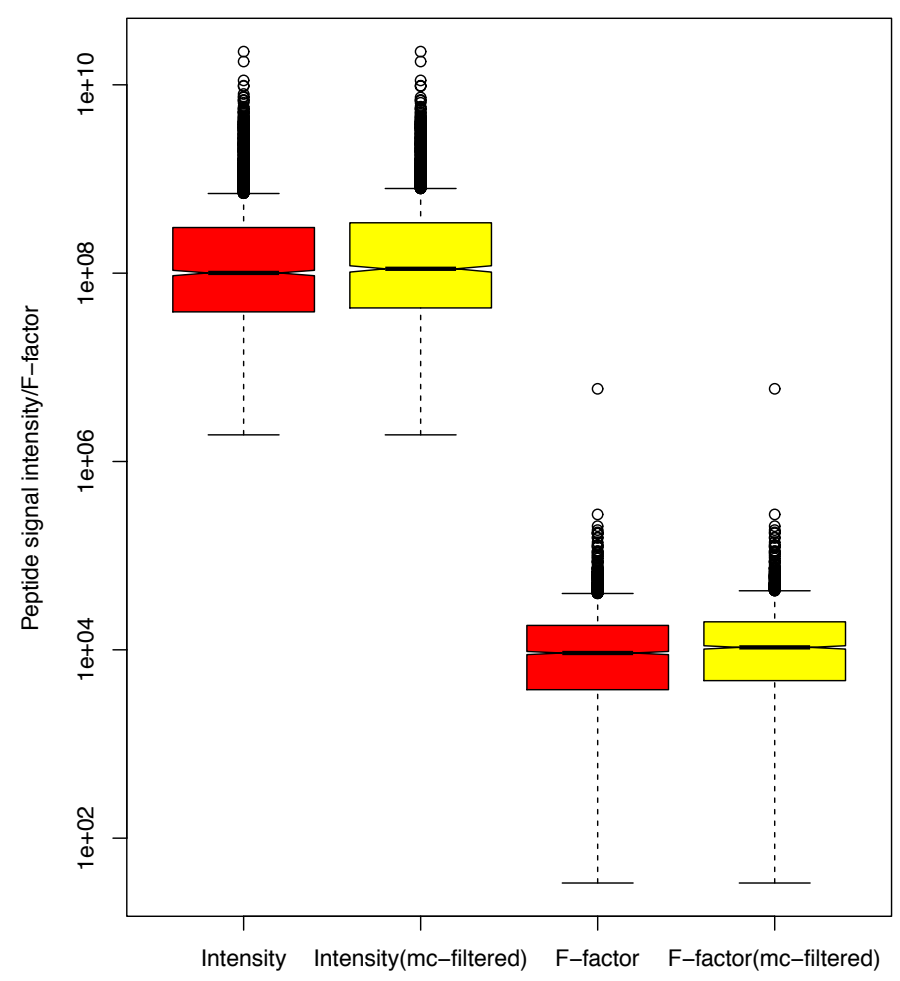

Supplementary Figure S4. Peptide signal intensity/F-factor distributions filtered by missed cleavage prediction. Peptide data from the $120 \mathrm{~min}$ gradient collected on the Q-Exactive HF was reanalysed, removing those peptides which were predicted to be strongly "missed cleaved" and therefore potentially strong candidates for signal loss owing to incomplete digestion from parent protein. We used our own prediction tool, McPred [1], to define the top $25 \%$ subfraction of observed peptides predicted to contain missed cleavage sites and filtered them out. The distributions of peptide signal intensities (red) and F-factors (yellow) are shown above, plotted as boxplots on a log scale. As can be seen, there is a small but significant increase in the apparent signal intensities and F-factors of the filtered subsets compared to all peptides, consistent with expectation. The effect appears to be relatively small however. 


\section{Supplementary Methods}

\section{Calculation of predicted retention time and coelution properties}

For the theoretical digests of organismal proteomes, peptide retention times were calculated using hydrophobicity index scales (HI) with the Sequence Specific Retention Calculator [2]. This was applied for the runs on the Q Exactive HF for the yeast and yeast- $E$. coli-spike-in proteome samples. First, $\mathrm{HI}$ scales were calculated for all experimentally detected peptides (FDR $<0.01$ ) for a given experimental set up and a linear calibration was obtained by plotting measured retention time in each experiment against the $\mathrm{HI}$ values. The linear model had an R-squared of 0.94 for both datasets, indicating a good fit. The retention times of all the theoretical peptides in both samples were then calculated by linear interpolation from the model's line of best fit. A small proportion of peptides with extreme hydrophobicity values were predicted to have negative or extremely large retention times: those were excluded and only peptides predicted to have a retention time within the experimentally observed range (i.e. between 7 and 120 minutes) were retained. The final retention time used for the analysis was the measured retention time where present, and the predicted time when measurement was not available.

The number of identified peptides classified as coeluting with a given peptide for the experimental runs was obtained by computing the number of peptides detected in at least one replicate within a 25 seconds window centred on that peptide. The number of theoretically coeluting peptides was calculated similarly, but using the predicted retention times.

\section{Supplementary References}

[1] Lawless, C.; Hubbard, S. J., Prediction of missed proteolytic cleavages for the selection of surrogate peptides for quantitative proteomics. Omics : a journal of integrative biology 2012, 16, (9), 449-56.

[2] O.V. Krokhin, V. Spicer, Peptide retention standards and hydrophobicity indexes in reversedphase high-performance liquid chromatography of peptides, Analytical chemistry, 81, 2009, 9522-9530. 